\title{
Antibiotic Susceptibility Pattern of Pseudomonas aeruginosa Isolated from Various Clinical Samples at a Tertiary Care Hospital
}

\author{
M. Wajid $^{1 *}$ and Shazia Naaz ${ }^{2}$ \\ Department of Microbiology, ESIC Medical College \& Hospital, \\ Sanath nagar, Hyderabad, India \\ *Corresponding author
}

\section{Keywords}

Pseudomonas aeruginosa, Antibiotic resistance, Levofloxacin

Article Info

\section{Accepted:}

15 December 2020 Available Online: 10 January 2021
Pseudomonas aeruginosa is a Gram-negative, rod-shaped and asporogenous bacterium. It has a pearlescent appearance and grape-like odour. P.aeruginosa grows well at $25^{\circ} \mathrm{C}$ to $37^{\circ} \mathrm{C}$, and its ability to grow at $42^{\circ} \mathrm{C}$ helps distinguish it from many other Pseudomonas species. It has been known to cause of variety of other infections like pneumonia, urinarytract infection, skin and soft-tissue infections, in severe burns and in infections among immunocompromised individuals. Objectives of the study is to isolate\& identify Pseudomonas aeruginosa \& other species (spp) from various clinical samples; to find out their distribution in various areas in the hospital; to study their antibiotic resistance pattern. It is a Lab based retrospective study conducted between the study period of April 2018March 2019 at the Department of Microbiology ESIC Medical College, Sanath Nagar, Hyderabad. A total of 10,988 samples were received at Department of Microbiology laboratory between the study period out of which 2488 samples were found to be culture positive. Pseudomonas aeruginosa \& spp was isolated in 142 samples (5.8\%) out of total 2488 samples. The prevalence of Pseudomonas aeruginosa \& spp. in our hospital setting was maximal in the In patient department (Wards \& ICU's). The strains isolated from out patient department were mostly found to be sensitive to most of the antibiotics. Highest resistance was noted in Pseudomonas aeruginosa \& Pseudomonas spp tolevofloxacin $(47.3 \%, 45.5 \%)$, ciprofloxacin $(45.7 \%, 39.1 \%)$, ceftazidime $(38.7 \%, 30.4 \%)$. Whereas least resistance was encountered with Tobramycin, Netilimycin \& piperacillin tazobactam followed by Carbapenams. Regular bacteriological identification and antimicrobial susceptibility surveillance $P$. aeruginosa is needed to identify the antimicrobial resistance pattern in the setting of our hospital.

\section{Introduction}

Pseudomonas aeruginosa is a Gram-negative, rod-shaped and asporogenous bacterium. It has a pearlescent appearance and grape-like odour. P. aeruginosa grows well at $25^{\circ} \mathrm{C}$ to $37^{\circ} \mathrm{C}$, and its ability to grow at $42^{\circ} \mathrm{C}$ helps distinguish it from many other Pseudomonas species. $P$. aeruginosa is a ubiquitous microorganism which has the ability to 
survive under a variety of environmental conditions. ${ }^{1}$

Pseudomonas aeruginosa is implicated in a wide variety of infections \& is gaining increasing resistance over the past few years. Pseudomonas aeruginosa has been implicated in various respiratory infections, especially cystic fibrosis, where it has tendency to persist due to formation of bio-films. It has been known to cause of variety of other infections like pneumonia, urinary-tract infection, skin and soft-tissue infections, in severe burns and in infections among immunocompromised individuals.

Independent risk factors have been identified for multi drug-resistant (MDR) or pan resistant P.aeruginosa infection like prior to use of antibiotics, history of $P$. aeruginosa infection or colonization within the previous year, length of hospital stay, being admitted as in-patient or in the intensive care unit (ICU), mechanical ventilation, malignant disease and history of chronic obstructive pulmonary disease . $^{2,3,4}$

The antibiotic resistance mechanisms include the acquisition of extended-spectrum $\beta$ lactamases, carbapenemases, aminoglycosidemodifying enzymes and $16 \mathrm{~S}$ ribosomal ribonucleic acid methylases. Mutational changes causing the up-regulation of multidrug efflux pumps, depression of ampC, modification of antimicrobial targets and changes in the outer membrane permeability barrier are also described. ${ }^{5}$

This study has been undertaken to check the antimicrobial susceptibility of Pseudomonas aeruginosa \&Pseudomonas species isolated from various samples in the hospital setting. The main objectives of this study include to isolate \& identify Pseudomonas aeruginosa \& other spp from various clinical samples (blood, urine, respiratory sample, pus, body fluids). To find out their distribution in various areas in the hospital and also to study their antibiotic resistance pattern.

\section{Materials and Methods}

\section{Study area}

Department of Microbiology ESIC Medical College, Sanath Nagar, Hyderabad.

\section{Study design}

It is a Lab based retrospective study conducted between the study period of April 2018 to March 2019. Permission from institutional ethics committee was obtained.

\section{Inclusion criteria}

All clinically significant Pseudomonas aeruginosa isolates will be included in the study.

Non-duplicate isolates were taken

\section{Exclusion criteria}

Repeated isolates from the same patient were excluded

A total of 142 Pseudomonas aeruginosa \& other Pseudomonas spp were isolated from different clinical specimens including wound swab/ pus, body fluids, urine, respiratory samples (bronchial wash, sputum, tracheal aspiration, throat pleural fluid) \& blood. The isolates were identified by colony morphology, Gram's staining and biochemical test according to standard laboratory test methods. The antibiotic susceptibility patterns of Pseudomonas isolates were analyzed by carrying out by disc diffusion method (Kirby-Bauer) in MullerHinton agar media according to the Clinical \& Laboratory Standards Institute (CLSI) 2019 
guidelines. ${ }^{6}$ Pseudomonas ATCC 27853 was used as control strain. The results of susceptibility test were categorized into susceptible and resistant.

\section{Statistical analysis}

The data was entered into excel spread sheet and statistical analysis was done by applying descriptive statistics to generate percentages.

\section{Results and Discussion}

A total of 10,988 samples were received at Department of Microbiology laboratory between the period of April 2018- March 2019 out of which 2488 samples were found to be culture positive. Pseudomonas aeruginosa \& spp was isolated in 142 samples (5.8\%) out of total 2488 samples.

The prevalence of Pseudomonas aeruginosa \& spp. in our hospital setting was maximal in surgery wards (46, 32.4\%), followed by Pulmonology ward $(28,19.7 \%)$, outpatient department $(17,12 \%)$ surgical ICU(13, $9.15 \%)$. The area wise distribution of Pseudomonas aeruginosa \& spp is depicted in Fig 1.

The maximum number of Psuedomonas spp isolates was from pus \& body fluids (51.4\%), followed by respiratory samples $(36.6 \%) \&$ urine $(7.04 \%)$. The findings of these are depicted in the table 1 .

Table.1 Shows the percentage of Pseudomonas isolates from various samples

\begin{tabular}{|l|l|}
\hline Specimen & No. of isolates $(\mathbf{n}=\mathbf{1 4 2})$ \\
\hline Urine & $10(7.04 \%)$ \\
\hline Pus \& body fluids & $73(51.4 \%)$ \\
\hline Respiratory samples (sputum, swab, tracheal aspirate, tissue) & $52(36.6 \%)$ \\
\hline Blood & $5(6.25 \%)$ \\
\hline CSF & $2(2.5 \%)$ \\
\hline
\end{tabular}

Table.2 Percentage Susceptibility of P.aeruginosa \& P.species from all clinical samples

\begin{tabular}{|r|l|c|c|c|c|}
\hline S.no & Medication & \multicolumn{2}{|c|}{ P.aeruginosa } & \multicolumn{2}{|c|}{ P.species } \\
\cline { 3 - 6 } & & S\% & R\% & S\% & R\% \\
\hline 1. & Amikacin (AMK) & 88.8 & 11.2 & 91.6 & 8.4 \\
\hline $\mathbf{2 .}$ & Ceftazidime(CAZ) & $\mathbf{6 1 . 3}$ & $\mathbf{3 8 . 7}$ & 69.6 & 30.4 \\
\hline 3. & Ciprofloxacin(CIP) & $\mathbf{5 4 . 3}$ & $\mathbf{4 5 . 7}$ & 60.9 & 39.1 \\
\hline $\mathbf{4 .}$ & Gentamicin(GEN) & $\mathbf{6 7 . 7}$ & $\mathbf{3 2 . 3}$ & 76.1 & 23.9 \\
\hline $\mathbf{5 .}$ & Imipenem(IPM) & 72.6 & 27.4 & 81 & 19 \\
\hline $\mathbf{6 .}$ & Levofloxacin(LVX) & $\mathbf{5 2 . 7}$ & $\mathbf{4 7 . 3}$ & 54.5 & 45.5 \\
\hline $\mathbf{7 .}$ & Meropenem(MEM) & 65.4 & 29.6 & 77.6 & 22.4 \\
\hline $\mathbf{8 .}$ & Piperacillin Tazobactam (TZP) & 87.2 & 12.8 & 81 & 19 \\
\hline $\mathbf{9 .}$ & Piperacillin(PIP) & 85 & 15 & 77.7 & 22.3 \\
\hline $\mathbf{1 0}$. & Tobramycin(TOB) & 91.8 & 8.2 & 88.8 & 11.2 \\
\hline $\mathbf{1 1}$. & Netilmicin(NET) & 90.1 & 9.9 & 75.6 & 24.4 \\
\hline 12. & Ticarcillin Clavulanate(TCC) & $\mathbf{8 6}$ & $\mathbf{1 4}$ & 81.8 & 18.2 \\
\hline
\end{tabular}


Fig.1 Area wise distribution of Pseudomonas aeruginosa \& spp in the hospital

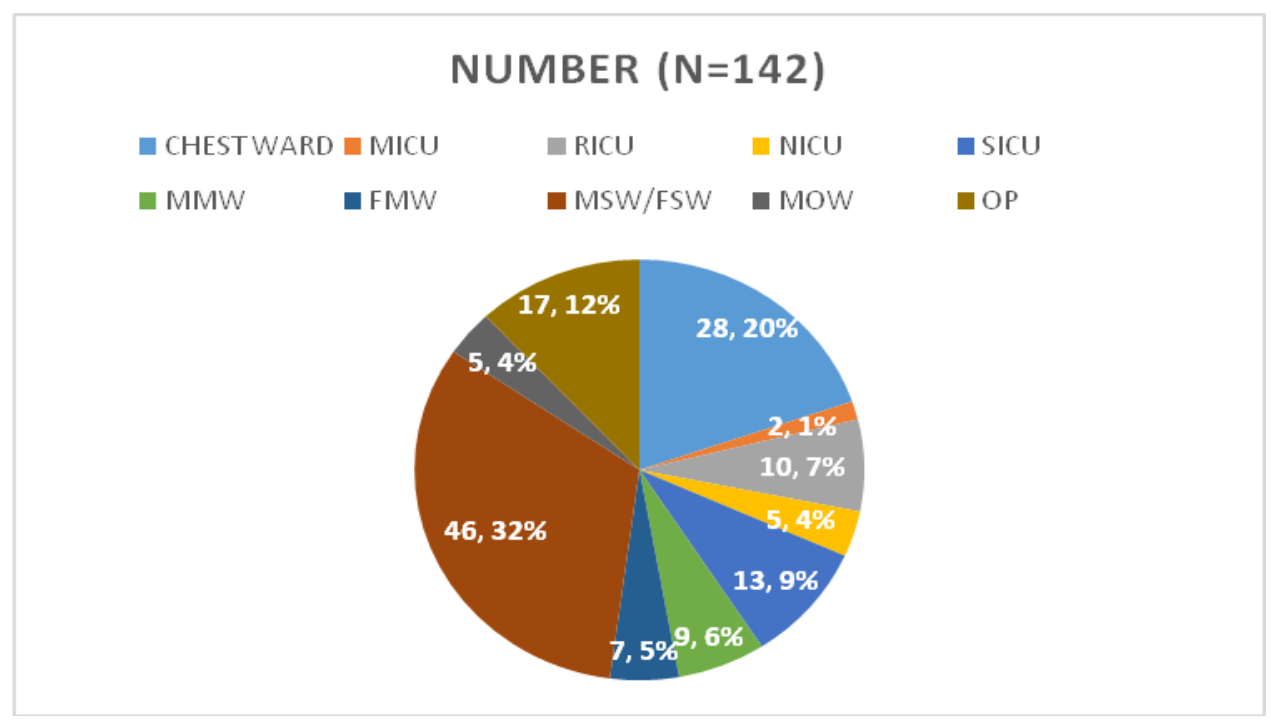

Highest resistance was noted in Pseudomonas aeruginosa \& Pseudomonas spp to levofloxacin $(47.3 \%, 45.5 \%)$,ciprofloxacin $(45.7 \%, 39.1 \%), \mathrm{c}$ eftazidime $(38.7 \%, 30.4 \%)$. Whereas least resistance was encountered with Aminoglycosides (Tobramycin, Netilimycin)\& piperacillin tazobactam followed by Carbapenams. The susceptibility patter of Pseudomonas aeruginosa \& spp is depicted in the table 2 .

Pseudomonas aeruginosa has emerged as a difficult to treat pathogen due to the various ways in which it evades the antimicrobial agents. The various knows ways in which they have developed resistance is due to the production of Extended betalactamase (ESBL), metalobetalactamses (MBL), Amp C.Out of 2488 culture positive samples.

Pseudomonas aeruginosa \& spp was isolated in 142 samples (5.8\%).In a study done by Sukesh, $\mathrm{K}$ et al the total number of Pseudomonas isolates were 32 out of 716 $(4.4 \%)^{7}$

Area wise distribution showed the prevalence of Pseudomonas aeruginosa \& spp. in our hospital setting in the In patient Department was maximal in surgery wards $(46,32.4 \%)$, followed by Pulmonology ward $(28,19.7 \%)$, surgical ICU $(13,9.15 \%)$.In the Out patient department the number of Pseudomonas strains isolated were $(17,12 \%)$

In a study done by Minu Kumari et al the prevalence of Pseudomonas spp.was maximal in neurosurgery ward $(590,31 \%)$, followed by surgery ward $(417,22 \%)$, surgical ICU (367, 19\%), neurosurgical ICU (284, 15\%), follow up outpatient department (163, 9\%), emergency department (39, 2\%) and orthopaedics ward $(37,2 \%) .{ }^{18}$

In this study, the maximum number of Psuedomonas spp isolates were from pus \& body fluids $(51.4 \%)$, followed by respiratory samples $(36.6 \%) \&$ urine $(7.04 \%)$.

In a study done by Jamshaid AK et al, most of the Pseudomonas spp isolates were from( pus $57.64 \%)^{8}$, followed by urine $24.2 \%$.

In a study by Sukesh $\mathrm{K}$ et al out of 100 clinical isolates of Pseudomonas aeruginosa, maximum isolates $(71 \%)$ are isolated from pus/swab followed by $16 \%$ from urine, $12 \%$ 
from sputum and 3\% from other samples. In both the studies urine was found to be the most common specimen after pus wherePseudomonas spp were isolated, in contrary to our study where respiratory samples were the next most common sample after pus.

In this study resistance to aminoglycoside amongst Pseudomonas aeruginosa \& Pseudomonas spp were as follows, gentamycin $(32.3 \%, 23.9 \%)$, and amikacin (11.2\% \& 8.4\%),tobramycin $(8.2 \%, 11.2 \%)$ respectively.Resistance to aminoglycosides was seen to be quite high in other parts of the world ,70.70\% by Lutfu Savas et al. and $69.86 \%$ by Agandi KM et al ${ }^{9,10}$ were documented, where as lower rate were reported from Pakistan $(6.73 \%$ by Nadeem et al. and $24 \%$ by Jamshaid et al. $)^{11,8}$ similar to our study.

Resistance amongst the flouroquinolone group was as follows to Pseudomonas aeruginosa \& Pseudomonas spp; ciprofloxacin $(45.7 \%, \quad 39.1 \%), \quad$ and levofloxacin $(47.3 \% \& 45.5 \%)$ respectively.

It has been observed in the study done by Hoque $\mathrm{MM}$ et al the resistance rate for ciprofloxacin was $54.04 \%$ which is similar to our study. ${ }^{12}$

Study done by Alyahawi A et al, documented a resistance of $34 \%$ to levofloxacin. ${ }^{19}$

Dash et all observed that in majority (77.7\%) of isolates were resistant, to more than 3 groups of antimicrobials, in which resistance to levofloxacin was found to be $36.1 \%$. $^{5}$

Slightly higher resistance to levofloxacin in our study may be due increased usage of this antimicrobial in our setting.

In a study done by Cetin Kilinic et al, it was determined that resistance to piperacillintazobactam was seen to be quite high $(57.9 \%) .{ }^{13}$ In our study, on the contrary it was found to be quite low $12.8 \%$ - \& $19 \%$ for Pseudomonas aeruginosa \& Pseudomonas spp respectively which is in accordance with other studies as determined by SENTRY Antimicrobial Surveillance Program (19972007). ${ }^{14}$ In this study it was concluded that, piperacillin/tazobactam remained a very active beta-lactam when tested in vitro against clinical isolates of $\mathrm{P}$. aeruginosa. ${ }^{14}$

In our study resistance to ceftazidime was found to be $38.4 \%$ for Pseudomonas aeruginosa isolates. Inan et al reported resistance against ceftazidime as $34 \%$

In a study carried out in Turkey the most effective antibiotics were carbapenems (imipenem and meropenem) and the resistance rates were detected as $15 \%$ and $20.4 \% .{ }^{9}$ Inan et al also reported resistance to Imipenem as $26 \%$ which is comparable to our study where we documented resistance in Pseudomonas aeruginosa strains to Imipenem $\&$ Meropenem to be $27.4 \%$ \& $29.6 \%$ respectively. ${ }^{16}$

Gamal F. Gad reported amikacin as the most active drug against $\mathrm{P}$. aeruginosa followed by meropenem, cefepime and fluoroquinolones. ${ }^{16}$ In an India study done by Muktikesh Dash et al, Imipenem, meropenem and piperacillin/tazobactam were most effective drugs observed which showed resistant rates of $6.4 \%, 8 \%$ and $11.3 \%$ respectively $^{17}$

In this present study, the most effective antimicrobials were the aminoglycoside group, followed by $\beta$-lactamase inhibitor combination (Piperacillin-Tazobactam Ticarcillin-clavulinate), Carbapenems, $\quad 3^{\text {rd }}$ generation cephalosporin and fluoroquinolones. 
Lower rate of resistance to aminoglycoside group of drugs, $\beta$-lactam $+\beta$ lactamase inhibitor combination (PiperacillinTazobactam Ticarcillin-clavulanate), Carbapenems may have been seen due to their lesser use in our set up.

In conclusion, regular bacteriological identification and antimicrobial susceptibility surveillance $P$. aeruginosa is needed to identify the antimicrobial resistance pattern in the setting of our hospital. Therefore each hospital must analyse their typical flora and analyse the antimicrobial susceptibility on a regular basis, so that there may be rational use of antimicrobial, decreasing the chances of spread of further antibiotic resistance in the community. Hospitals harbour many antimicrobial resistant strains, so utmost care must be taken to further enhance the resistance pattern in our health care systems by prudent antimicrobial stewardship.

\section{References}

1. Yi-Wei Tang, Dongyou Liu, Ian Poxton, Joseph Schwartzman, max Sussman. Molecular Medical Microbiology. Chapter 41 - Pseudomonas aeruginosa. Volume 2, 2015, Pages 753-767.

2.Ohmagari N, Hanna H, Graviss L, Hackett B, Perego C, Gonzalez V, et al. Risk factors for infections with multidrugresistant Pseudomonas aeruginosa in patients with cancer. Cancer 2005; 104:205-12.

3.Aloush V, Navon-Venezia S, Seigman-Igra Y, Cabili S, Carmeli Y. Multidrugresistant Pseudomonas aeruginosa: Risk factors and clinical impact. Antimicrobial Agents Chemotherapy 2006; 50:43-8.

4.Arruda EA, Marinho IS, Boulos M, Sinto SI, Caiaffa HH, Mendes CM, et al. Nosocomial infections caused by multiresistant Pseudomonas aeruginosa. Infect Control Hosp Epidemiology 1999; 20:620-3.

5.Dash M, Padhi S, Narasimham MV, Pattnaik S. Antimicrobial resistance pattern of Pseudomonas aeruginosa isolated from various clinical samples in a tertiary care hospital, South Odisha, India. Saudi J Health Sci 2014; 3:15-9

6.Clinical \& Laboratory Standards Institute (CLSI) 2019 guidelines.

7.Sukesh, K. and Prakash Vincent, S. G. $2017 . \quad$ "Microbiological characterization and antibiotic sensitivity pattern of Pseudomonas aeruginosa isolated from clinical samples", International Journal of Development Research, 7, (10), 16428-16431.

8.Jamshaid AK, Zafar I, Saeed UR et al. Prevalence and resistance patterns of Pseudomonas aeruginosa against various antibiotics. Pak J. Pharma. Sci. 2008; 21:311-5.

9.Savas L, Duran N, Savas N et al. The prevalence and Resistance Patterns of Pseudomonas aeruginosa in Intensive Care Units in a University Hospital. Turkey Journal of Medical Science. 2005; 35:317-22.

10. Angadi KM, Kadam M, Modakn MS et al. Detection of Antibiotic Resistance in Pseudomonas aeruginosa Isolates With Special Reference to Metallo $\beta$ Lactamases From a Tertiary Care Hospital In Western India. International Journal of Microbiology Research. 2012; 14 (7):295-8.

11.Raja NS, Singh NN. Antimicrobial susceptibility pattern of clinical isolates of Pseudomonas aeruginosa in a tertiary care hospital. J Microbiol Immunol Infect. 2007 Feb;40(1):45-9. PMID: 17332906

12.Hoque MM1, Ahmad M2, Khisa S3, Uddin MN4, Jesmine R.Antibiotic 
Resistance Pattern in Pseudomonas Aeruginosa Isolated from Different Clinical Specimens.JAFMC Bangladesh. Vol 11, No 1 (June) 2015.

13.Kılınç Ç, Güçkan R, Çepni M, Aydın O, Çatakoğlu AH. Antibacterial Resistance in Pseudomonas aeruginosa Strains Isolated from Various Clinical Samples. Eur J Health Sci 2015;1(3):96-100.

14.Antipseudomonal activity of piperacillin/tazobactam: more than a decade of experience from the SENTRY Antimicrobial Surveillance Program (1997-2007).

15.Inan D, Ögünc $\mathrm{D}$, Günseren $\mathrm{F}$ et al. The resistance of Pseudomonas aeruginosa strains isolated from nosocomial infections against various antibiotics. Mikrobiyol Bult 34: 255-60, 2000

16. Gad GF, El-Domany RA, Zaki S, Ashour
HM. Characterization of Pseudomonas aeruginosa isolated from clinical and environmental samples in Minia, Egypt: prevalence, antibiogram and resistance mechanisms. J Antimicrob Chemother. 2007 Nov; 60(5):1010-7. doi: 10.1093/jac/dkm348. Epub 2007 Sep 29. PMID: 17906321.

17.Dash M, Padhi S, Narasimham MV, Pattnaik S. Antimicrobial resistance pattern of Pseudomonas aeruginosa isolated from various clinical samples in a tertiary care hospital, South Odisha, India. Saudi J Health Sci 2014; 3:15-9.

18.Kumari M, Khurana S, Bhardwaj N, Malhotra R,Mathur P. Pathogen burden \& associated antibiogram of Pseudomonas spp. in a tertiary care hospital of India.Indian J Med Res. 2019 Feb; 149(2): 295-298.

\section{How to cite this article:}

Wajid, M. and Shazia Naaz. 2021. Antibiotic Susceptibility Pattern of Pseudomonas aeruginosa Isolated from Various Clinical Samples at a Tertiary Care Hospital. Int.J.Curr.Microbiol.App.Sci. 10(01): 2308-2314. doi: https://doi.org/10.20546/ijcmas.2021.1001.267 